SECTION 21. Pedagogy. Psychology. Innovations in the field of education.

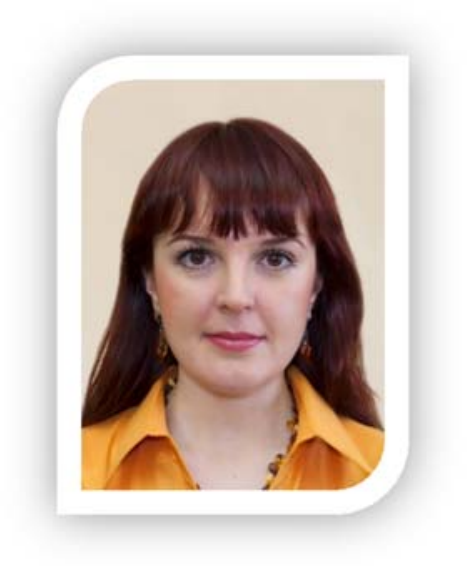

Natalia Alexandrovna Nastashchuk

Assoc. Prof., Doctor of Philosophy in Pedagogy, Department "Informatics, applied mathematics and mechanics" Omsk State Transport University, Russian Federation, Omsk nat_lion@mail.ru

\title{
SIGNIFICANCE OF THREE-VALUED LOGIC IN INFORMATICS FOR STUDENTS FROM TECHNICAL UNIVERSITIES
}

\begin{abstract}
The article is considered application of Three-Valued Logic in informatics on the whole. It reveals significance of Three-Valued Logic for students from technical universities who will do some research in engineering area as well as apply it in a professional engineering activity.
\end{abstract}

Key words: Three-Valued Logic, engineering education.

$\mathrm{XX}$ and XI century are marked by a progressive increase of protest against the twodigit. It is presented into two ways. The first one is a problem of up-to-date mathematical logic that closely associated with the lack of presentation of fundamental logical relations in double-valued algebraic objects. There are intuitionistic mathematics rejection of the excluded middle; Lewis's attempts and then Ackermann's attempts for overcoming "paradoxes" of material implication; an invention of Lukasiewicz's Three-Valued Logic, Reichenbach's assumption about Three-Valued Logic microcosm (Quantum Mechanics), general increase of activity in the field of Multi-Valued Logic and, finally, fuzzy sets introduced by Lotfi A. Zadeh.

The increase productivity of digital devices is one of the main problems in modern circuit techniques [6]. For example, the achieved clock frequency is close to the limit, not suitable for parallelization of any problem. The developers of microprocessor technology are interested in Multi-Valued Logics, particularly, in ternary implementation of microprocessor technology ${ }^{1}$ and nanotechnology. Nowadays Ternary Logic is increasingly used in design of digital circuits, electronics and telecommunications.

So, researchers' attention again turn to the schemes which use Three-Valued Logic. More over Three-Valued Logic may be used in the development of computer intellectual functions and artificial intelligence. In Brusentsov's judgment two-digit mathematical logic does not correspond to common sense: "the law of the excluded middle" cuts other conclusions except “Truth" and "Non-Truth". But the process of man's cognition of reality can not be reduced to the dichotomy of "Yes /No". Brusentsov N. P. states that an intellectual

${ }^{1}$ There are such companies as IBM, Motorola and Texas Instruments who lead research work based on silicon-germanium alloys. In this case, it is possible to realize a digital integrated circuit operating with three or more signal levels. 
computer should be ternary $[1,2,3]$. Some researchers including Brusentsov N. P. ${ }^{1}$ believe that the use of Ternary Logic will approach closer to solving problems of artificial intelligence [4]. In this connection Three-Valued Logic should be learned in informatics by students from technical universities.

It is proposed to include TVL in training informatics students from technical universities. This course of informatics is based on the suggested text-book of informatics for technical universities [7].

The theme "Informatics. Description of information" should include the basic concepts of Ternary Logic as well as its application and significance in management of computing machines.

Three-Valued Logic (also Trivalent, Ternary, Trinary Logic, or Trilean, sometimes abbreviated 3VL) is one of several Many-Valued Logic systems in which there are three truth values indicating "True", "False" and some indeterminate third value "Unknown" (also mean "Undefined" or "Neither"). Conceptual forms and basic ideas of 3VL were initially created by Jan Łukasiewicz and C. I. Lewis. 3VL is historically the first Three-Valued Logic (TVL). It is an extension of Boolean Logic (Two-Valued Binary Logic) or Boolean Algebra.

It is necessary to point out that there are crisp mathematical 3VL and fuzzy 3VL. Crisp mathematical 3VL has three crisp values $(0,1,2),(-1,0,+1),(0,1 / 2,1)$. Fuzzy 3VL is a particular case of Fuzzy Logic with three values: one, two or all three values are fuzzy. For example, the values of fuzzy 3VL with one crisp and with two fuzzy values are "Less", "Equally", "More” etc [3].

Logic operating three values corresponds to ternary or symmetric ternary numerical notation. It is suitable for data representation. It is represented like a system with the base which is equal to the Euler number 2.71. The nearest integer to this number it is three. Hence, the ternary system is optimal numerical notation with the integer base [5]. In data transmission a ternary signal carries information in 1.6 times more than a binary signal. So, computer storage device with ternary cells stores data in 1.6 times more than digital computer storage device with the same number of cells [1].

Ternary numerical notation (having three digits, for example, $+1,0$ и -1 ) gives the following advantages. In this system negative numbers are represented like positive numbers: there is no need to allocate a special place in the sign bit. Operations with negative numbers are carried out without preliminary operations: reduction to reverse or additional code as in the case of operations with negative numbers in the binary system. In addition, ternary numbers are simpler compared and rounded than digital numbers.

Three types of signals formed by a basic unit of a ternary computer are called trit. Information capacity of trit is equal to about 1.5 bytes. The ternary computer processes more data per unit time than a binary computer.

In the theme "Information engineering" it is supposed to include some information about the ternary computer and the base of design arithmetic circuits in TVL (ternary technologies).

Brusentsov N. P. is a main constructor of the SETUN ternary computer in Russia. It was developed in 1958 by him and his team at the Moscow State University. SETUN is based on TVL $(-1,0,1)$ which completely distinguishes it from the binary computer. While TwoValued Binary Logic just allows two states yes (1) | no (0), TVL has three different logical states: yes (1) | no (-1) | both (0). Western scientists tried to create the ternary computer in the following years but never succeeded.

Students should expand their knowledge in the history of computing machines. For example, the work [4] deals with the production and representation of knowledge, the

${ }^{1}$ Brusentsov N. P. is a developer of the first practical computer running a three-value system. 
historical and ideological aspects of technological development. And it aims to create an understanding of the intertwining of current technological and social developments using a historical perspective. The book makes original Russian sources available in English and German language and is accompanied by an original essay investigating into the relations between the "communist" Easts and capitalist Wests technological development. While the book aimes to provide a basis for further research, the artistic work tries to involve visitor and artist into what can not be expressed within the realm of theoretical discourse.

Trait is a minimum addressable unit of memory in the ternary computer. It is equal 6 trits and possesses the value from -364 to 364. Operation with a range of negative values is a peculiarity which distinguishes trait from bitsbyte. The values of bitsbyte cover the range from 0 to 255.

TVL has several advantages in comparison with Double-Digit Logic. But although most modern computers are based on the two-digit representation of numbers, there are thanks to the capabilities of semiconductor technology. So, new perspectives are opened up for the ternary computers. For example, a creation of a photon computer is a perspective trend in the field of the development of the ternary computer. A computer operating three values can be created. It will be high-performance and resistant to electro-magnetic interference.

\section{References:}

1. Brusentsov N. P. Experience in the development of the ternary computer. - Herald of the Moscow University. Serial Number. I, Mathematics, mechanucs, 1965, № 2, P. 3948.

2. Brusentsov N. P. Beginning of informatics. - Moscow: Fund "New millennium”, 1994.

3. Brusentsov N. P., Derkach A.Yu. Three-valued logic, fuzzy sets and probability theory. - // Software systems and tools. Thematic collection Number 2. - Moscow: Faculty MGU, 2001, P. 88-91.

4. Hunger F. SETUN. An Inquiry to the Soviet Ternary Computer (Eine Recherche über den sowjetischen Ternärcomputer) Institut für Buchkunst Leipzig, 2008.

5. Phomin S.V. Numerical notations. - Moscow: Science, 1987.

6. Sheng L., Yong-Bin K., and Lombardi F. CNTFET-Based Design of Ternary Logic Gates and Arithmetic Circuits //IEEE Trans. Nanotechnology. 2011. V. 10. No. 2. P. 217-225.

7. Siretskey G.A. Informatics. Fundamental course. Volume I. The base of informational and computer engineering. - St. Petersburg: BHV-Peterburg, 2005. 